\title{
Durabilidade natural do estipe de pupunha (Bactris gasipaes Kunth, Arecaceae) II: Insetos.
}

\author{
Raimunda Liege Souza de ABREU ${ }^{1}$, Maria Aparecida de JESUS ${ }^{1}$
}

\begin{abstract}
RESUMO
Neste trabalho estão apresentados os resultados da durabilidade natural do estipe (madeira) de Bactris gasipaes Kunth (pupunha), quando submetido ao ataque de insetos xilófagos, em ensaios em ambiente florestal e urbano. Foram utilizados dez palmeiras, cinco com espinhos e cinco sem espinhos, de plantios da Fazenda Experimental da Universidade Federal do Amazonas, localizada no km 40 da rodovia Manaus-Boa Vista (BR 174). De cada uma das palmeiras foram cortados três discos de aproximadamente $30 \mathrm{~cm}$ de espessura, retirados da base, do meio e do topo. No ambiente florestal, os discos foram distribuídos aleatoriamente, em área próxima ao plantio, no espaçamento de $0,5 \mathrm{~m}$, permanecendo durante 18 meses, período no qual foram efetuadas seis inspeções trimestrais para avaliar o grau de deterioração e coleta de insetos. Para o ensaio em condição urbana, os discos foram secionados axialmente para a retirada da medula e distribuídos aleatoriamente, nas posições côncava e convexa, sobre uma estrutura de madeira, localizada no Campus do INPA em Manaus, e inspecionados bimestralmente por um ano. Os resultados do ensaio no ambiente florestal indicaram que a maioria dos discos foi deteriorada por térmitas e a vida útil da base foi em torno de 18 meses, a do meio e do topo em torno de 15. As principais espécies de cupins foram: Heterotermes tenuis (Hagen) (Rhinotermitidae) responsável pela deterioração da parte basal, mediana e o topo; Nasutitermes similis Emerson (Termitidae) que infestou a região da base e do meio; Anoplotermes sp. (Termitidae) e Nasutitermes tatarandae (Holmgren) (Termitidae) responsáveis pela infestação da parte mediana do estipe. No ambiente urbano, o principal responsável pela deterioração das amostras foi o besouro Dinoderus bifoveolatus Wollston (Bostrichidae), e em seguida, o térmita N. similis.
\end{abstract}

PALAVRAS-CHAVE

Bactris gasipaes, biodeterioração, Coleoptera, Isoptera.

\section{Natural durability of Bactris gasipaes Kunth (peach palm, Arecaceae) stipe II: Insects.}

\begin{abstract}
The durability of the stipe of Bactris gasipaes Kunth (Peach palm) when under attack by xylophage insects, is evaluated in the present paper, through forest and urban environment field tests. Five palms with spines and five without spines from the Tabatinga population (Putumayo landrace) were used in the experiment. Disks approximately $30 \mathrm{~cm}$ thick were taken from the butt, middle and top portions of each palm and distributed at random in a forest area near the

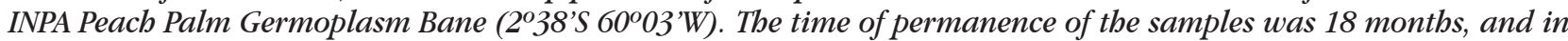
this period the disks were checked every three months to evaluate the degree of deterioration and collection of insects. In the urban condition, the same procedure was adopted, however each disk was halved to remove the centre and each half was distributed at random, one in a concave and the other in a convex position, on a wooden framework situated in the INPA's campus, Manaus, and checked bimonthly for one year. The results in the forest condition indicated that most of the disks were deteriorated by termites, and useful life of the butt portions varied around 18 months, the middle and the top around 15. The main species of termites were: Heterotermes tenuis (Hagen) (Rhinotermitidae) attacked the butt, middle and the top parts; Nasutitermes similis Emerson (Termitidae) that infested the area of the base and of the middle; Anoplotermes sp. (Termitidae) and Nasutitermes tatarandae (Holmgren) (Termitidae) attacked the medium part of the stipe. In the urban condition, the beetle Dinoderus bifoveolatus Wollston (Bostrichidae), was the most responsible for the deterioration of the samples, followed by the termite $\mathrm{N}$. similis.
\end{abstract}

\section{KEY WORDS}

Bactris gasipaes, biodeterioration, Coleoptera, Isoptera.

\footnotetext{
${ }^{1}$ Coordenação de Pesquisas de Produtos Florestais/Instituto Nacional de Pesquisas da Amazônia, Av. André Araújo 2936, Petrópolis, Caixa Postal 478, Manaus, AM, Brasil. E-mail: raiabreu@inpa.gov.br
} 


\section{ACTA \\ AMAZONICA}

DURABILIDADE NATURAL DO ESTIPE DE PUPUNHA

(Bactris gasipaes KUNTH, ARECACEAE) II. INSETOS

\section{INTRODUÇÃO}

Estudos de laboratório e de campo para realizar levantamentos de insetos xilófagos e avaliar a durabilidade de espécies florestais da região Amazônica ao ataque de insetos têm sido realizados pela Coordenação de Pesquisas de Produtos Florestais do Instituto Nacional de Pesquisas da Amazônia, dentro do contexto da caracterização tecnológica, com vistas a um melhor aproveitamento da madeira (INPA/CPPF, 1991; 1993; Abreu \& Bandeira, 1992; Jesus et al., 1998; Abreu \& Silva, 2000).

O teste de campo é um dos parâmetros utilizados para avaliar a resistência e a vida útil de espécies arbóreas, quando submetidas ao ataque de insetos e de outros organismos. Esses testes, realizados em condições ambientais, sejam na floresta ou em ambiente urbano, envolvendo condições de temperatura e umidade diferentes, servem de suporte para avaliar em que situação determinada espécie arbórea poderá ser utilizada.

A palmeira Bactris gasipaes Kunth (pupunha) é encontrada principalmente na Amazônia Ocidental e no sul de América Central (Clement, 1988), onde seus frutos e palmito são muito utilizados na dieta da população, sendo inclusive objeto de estudos nessas regióes (Clement \& Mora Urpí, 1987; Clement \& Arkcoll, 1991; Yuama, 1997). É uma espécie doméstica de rápido crescimento e, quando da renovação do plantio, seu estipe (tronco) é descartado (Araújo, 1991; Gomes, 1993). Em virtude disso, houve o interesse na caracterização tecnológica do estipe, visando o seu aproveitamento, inclusive com perspectivas para uso na confecção de pequenos objetos, instrumentos musicais e móveis (Lobato et. al., 1999). Desta maneira, agrega-se valor a este material, ao mesmo tempo em que contribui com as perspectivas atuais do aproveitamento de resíduos florestais.

Apesar dos estudos realizados, são escassos os conhecimentos a respeito de insetos que atacam a parte lenhosa da pupunheira. Neste contexto, o presente trabalho teve como objetivos o levantamento de insetos, especialmente besouros e cupins, e avaliação da durabilidade natural do estipe de $B$. gasipaes quando submetido ao ataque desses organismos em ambientes florestal e urbano.

\section{MATERIAL E MÉTODOS}

O levantamento de insetos e a avaliação da durabilidade natural do estipe de $B$. gasipaes foram feitos por meio de ensaios em ambiente florestal e urbano, com dez árvores da pupunha, sendo cinco da variedade com espinhos e cinco da sem espinhos da população Tabatinga (raça Putumayo). $\mathrm{O}$ tronco dessas palmeiras foi secionado na base, no meio e no topo e de cada uma dessas partes foram cortados dois discos de aproximadamente $30 \mathrm{~cm}$ de comprimento, tirados em altura equivalente de cada um dos troncos.

O ensaio em ambiente florestal foi realizado na área do Banco Ativo de Germoplasma de Pupunha, localizado no Km 40 da rodovia Manaus-Boa Vista ( $\left.2^{\circ} 38^{\prime} \mathrm{S} 60^{\circ} 03^{\prime} \mathrm{W}\right)$, cujo solo foi descrito por Falesi (1971) e por Ranzani (1980), como latossolo amarelo, com textura muita pesada em todo o perfil e integrada por solos profundos, bastante desgastados, fortemente ácidos e bem drenados, com teor de argila acima de $70 \%$ no horizonte $\mathrm{B}$; o clima, segundo a classificação de Köpen, é do tipo Ami "A" (clima tropical chuvoso), com temperatura média anual de $26,7^{\circ} \mathrm{C}$, índice pluviométrico anual na ordem de $2101 \mathrm{~mm}$ e média anual da umidade relativa de 84\% (Vieira \& Santos, 1987).

Para a realização deste ensaio, os discos foram distribuídos aleatoriamente no solo da floresta secundária, próximo ao plantio das pupunheiras no espaçamento de $0,5 \mathrm{~m}$. O tempo de permanência das amostras neste ambiente foi de 18 meses (julho de 1995 a janeiro de 1997) e neste período, a cada três meses, foram efetuadas coletas de insetos e inspeções visuais (Jesus \& Abreu, 2002) para avaliar o nível de infestação ou deterioração dos discos, de acordo com os critérios relacionados na Tabela 1 , descritos por Serpa (1982). Para melhor compreensão do nível de deterioração, uma vez que os insetos infestaram diferentes partes dos discos ensaiados, tornou-se necessária a divisão dos mesmos em revestimento, medula (tecido esponjoso) e madeira (parte lignificada) .

O ensaio em ambiente urbano foi realizado no campus do Instituto Nacional de Pesquisas da Amazônia em Manaus, em uma área com incidência homogênea dos raios solares. Para este ensaio, os discos foram secionados axialmente para a retirada do tecido esponjoso, porque se pretendia estudar a utilização da parte lignificada em ambiente externo e interno e também como telha, assoalho, dentre outros. Em seguida, foram distribuídos aleatoriamente nas posições convexa e côncava, sobre três estruturas de madeira de $70 \mathrm{~cm}$ de altura, $20 \mathrm{~cm}$ de largura e $2 \mathrm{~m}$ de comprimento. A cada dois meses, durante o período de setembro de 1996 a agosto de 1997 , foram efetuadas coletas de insetos e realizadas inspeções, cujos critérios foram os mesmos adotados no ensaio em ambiente florestal (Jesus \& Abreu, 2002).

Tabela 1 - Classificação do nível de deterioração dos discos das palmeiras em ensaio de campo.

\begin{tabular}{ccl}
\hline \hline Nota & Índice de Comportamento (\%) & \multicolumn{1}{c}{ Nível de deterioração } \\
\hline 0 & 100 & Ausência de sintoma de deterioração \\
1 & 90 & Deterioração superficial por fungos ou térmitas \\
2 & 70 & Deterioração evidente, porém moderada causada por fungos ou térmitas \\
3 & 40 & Deterioração intensa, ou colonização intensa por fungos e térmitas \\
4 & 0 & Perda total da resistência \\
\hline \hline
\end{tabular}

Fonte: Serpa (1982) 


\section{ACTA \\ AMAZONICA}

Os cupins coletados, tanto no ambiente florestal como no urbano, foram identificados pelo Dr. Reginaldo Constantino da Universidade de Brasília e os besouros por comparação com exemplares previamente identificados da Coleção Sistemática de Invertebrados, do Instituto Nacional de Pesquisas da Amazônia (INPA).

\section{RESULTADOS}

Durabilidade natural de $B$. gasipaes em ambiente florestal

Em ambiente florestal, os discos foram infestados por cupins desde a primeira inspeção até a última e aparentemente não houve distinção de ataque entre as amostras da variedade com espinhos e as da sem espinhos. Heterotermes tenuis (Hagen) (Rhinotermitidae) foi o principal responsável pela deterioração da maioria dos discos. Infestou os discos retirados de todas as alturas do estipe e foi encontrado principalmente durante os primeiros doze meses do experimento, iniciando com ataque superficial (nota 1) a moderado (nota 2) nos três primeiros meses. Entre nove e doze meses, chegou à deterioração total (nota 4) das amostras. Em algumas amostras da base e do meio, a deterioração total só foi atingida aos 15 meses. Nasutitermes similis Emerson (Termitidae) atacou os discos da base e do meio da pupunheira, enquanto que Nasutitermes tatarandae (Holmgren) (Termitidae) e Anoplotermes sp. (Termitidae) demonstraram preferência pelos discos da altura mediana. $N$. similis iniciou seu ataque após o terceiro mês de exposição dos discos no campo e as duas últimas espécies, após o sexto mês, permanecendo nos discos até o final do experimento.

Os térmitas foram encontrados tanto na madeira (parte lignificada) quanto na medula dos discos, demonstrando a alta suscetibilidade destas partes, com destaque para a medula que foi totalmente degradada. As partes da base e do meio dos estipes foram infestadas pelos térmitas logo nos três primeiros meses de exposição no campo, e eles permaneceram mais tempo nessas partes. Nos discos do ápice, a infestação ocorreu após o terceiro mês, e a permanência dos cupins nos mesmos foi menor (Tabela 2).

Foi observada também, nos primeiros três meses do experimento, a presença de besouros da família Curculionidae nos discos das três alturas do estipe, porém o dano foi superficial (nota 1).

Durabilidade natural de B. gasipaes em ambiente urbano

Nesse ambiente, aparentemente também não houve diferenças entre as variedades testadas. Os primeiros a se estabelecerem nas amostras foram os besouros (Tabela 3), representados principalmente por Dinoderus bifoveolatus Wollaston (Bostrychidae), encontrado nas quatro primeiras inspeções, feitas no período de oito meses. O ataque deste inseto foi aumentando gradativamente até ocasionar, em alguns discos, deterioração total (nota 4), principalmente na parte lignificada. No entanto, as amostras do ápice foram menos deterioradas por este inseto. Outro ponto a ser considerado é que, apesar deste inseto ter sido encontrado tanto nos discos na posição côncava quanto na convexa e no revestimento e na parte lignificada, a maior ocorrência do mesmo foi registrada nas amostras expostas na posição côncava.

Concomitante ao D. Bifoveolatus, foi encontrado Minthea rugicollis Walk (Lyctidae) em algumas amostras, de todas as alturas da pupunheira, sendo o ataque moderado (nota 2). Na primeira inspeção, foram encontrados Hypotbenemus eruditus Westwood e Xyleborus ferrugineus Fabricius, pertencentes à família Scolytidae, com ataque superficial.

Foi observada a presença do térmita $N$. Similis, que se estabeleceu a partir dos 10 meses de exposição dos discos, tendo uma passagem efêmera, porém ativa, pois sua permanência por apenas dois meses contribuiu para a deterioração total (nota 4) de muitas das amostras da pupunheira expostas nas duas posições.

\section{DISCUSSÃO}

A espécie $H$. tenuis, principal responsável pela deterioração dos discos da pupunheira na floresta, tem sido encontrada na Amazônia em área florestal e urbana, em madeira sadia a pouco ou medianamente decomposta, pois é considerado xilófago verdadeiro (Bandeira et al., 1989; Matias \& Abreu, 1999). Em levantamento realizado em madeiras de edificações em Belém, no Estado do Pará, Bandeira et al. (1989) encontraram esta espécie atacando pisos de madeira e prateleiras velhas, porém sem se constituir um problema sério, pois segundo os autores, a referida espécie prefere madeira muito úmida, que esteja em contato com o solo e sob ação de chuvas, o que vem confirmar o fato dela ter sido a primeira espécie a atacar a parte lignificada da pupunheira e permanecer por um longo período.

As demais espécies, principalmente as do gênero Nasutitermes, fazem parte do grupo de insetos considerados como os mais importantes decompositores de madeiras em florestas tropicais (Martius, 1994). Segundo este autor, elas são encontradas em todos os ecossistemas florestais da Amazônia e representam 25\% da população de térmitas dessa região. Alimentam-se praticamente de todos os produtos vegetais, incluindo madeira dura ou mole, seca ou úmida, trabalhada ou não, folhas, gramíneas e outros substratos (Bandeira \& Macambira, 1988; Bandeira et al., 1989; Martius, 1994). Algumas espécies desse gênero também têm preferência por espécies de madeira e também por diferentes estágios de decomposição (RodriguezBustamante \& Martius, 1998). Neste trabalho, foi observado que os Nasutitermes começaram o ataque após algum tempo de exposição dos discos na floresta. Já no estudo realizado por Rodriguez-Bustamante \& Martius (1998), quando três espécies florestais da várzea, com diferentes estágios de decomposição, foram submetidas ao ataque de 


\section{ACTA \\ AMAZONICA \\ DURABILIDADE NATURAL DO ESTIPE DE PUPUNHA \\ (Bactris gasipaes KUNTH, ARECACEAE) II. INSETOS}

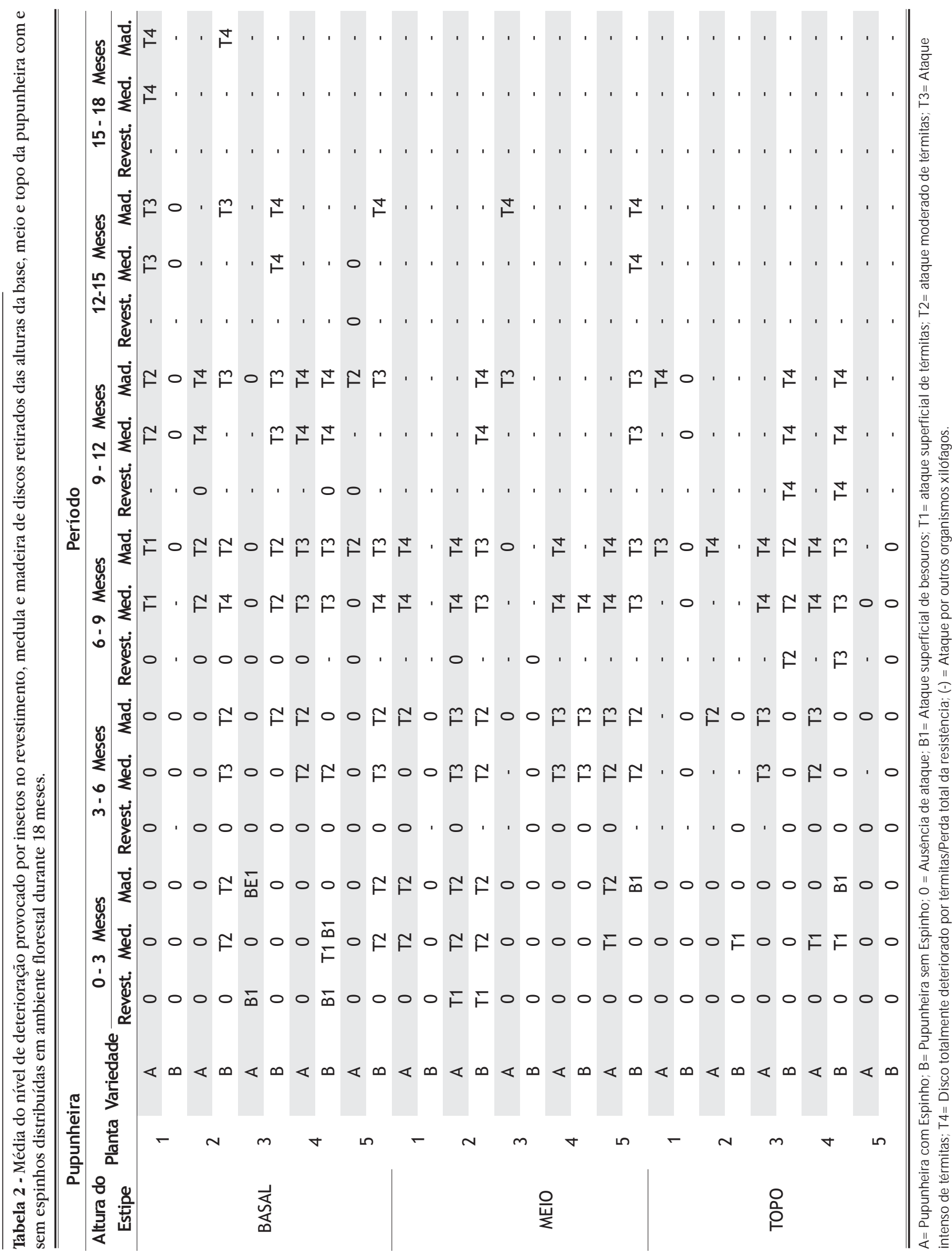




\section{ACTA AMAZONICA}

Tabela 3 - Média do nível de deterioração provocado por insetos no revestimento e madeira de discos retirados da base, meio e topo da pupunheira com e sem espinhos distribuídos em ambiente urbano durante 12 meses.

\begin{tabular}{|c|c|c|c|c|c|c|c|c|c|c|c|c|c|c|}
\hline \multicolumn{3}{|c|}{ Pupunheira } & \multicolumn{12}{|c|}{ Período } \\
\hline \multirow{2}{*}{$\begin{array}{l}\text { Altura do } \\
\text { Estipe }\end{array}$} & \multirow{2}{*}{ Planta } & \multirow{2}{*}{ Variedade } & \multicolumn{3}{|c|}{0 - 2 Meses } & \multicolumn{3}{|c|}{ 2- 4 Meses } & \multicolumn{3}{|c|}{ 4- 6 Meses } & \multicolumn{3}{|c|}{6 - 8 Meses } \\
\hline & & & Revest. & Med. & Mad. & Revest. & Med. & Mad. & Revest. & Med. & Mad. & Revest. & Med. & Mad. \\
\hline \multirow{10}{*}{ BASAL } & \multirow{2}{*}{1} & A & 0 & 0 & 0 & B1 & B1 & B3 & B1 & B3,5 & - & - & - & - \\
\hline & & B & 0 & 0 & 0 & 0 & - & B1 & B1 & B3 & - & - & T4 & T4 \\
\hline & \multirow{2}{*}{2} & A & 0 & B1 & 0 & B2, 5 & B1 & B3 & B1 & B4 & - & - & - & - \\
\hline & & B & 0 & B1 & 0 & B1,5 & B1 & $B 2,5$ & B1 & B2, 5 & - & $\mathrm{T} 2$ & $\mathrm{~T} 4$ & $\mathrm{~T} 4$ \\
\hline & \multirow{2}{*}{3} & A & 0 & B2 & 0 & B2 & B1 & B3,5 & - & B3,5 & - & - & T4 & T4 \\
\hline & & B & 0 & B3 & 0 & B3,5 & B1 & $\mathrm{B} 3,5$ & B2 & $\mathrm{B} 3,5$ & - & - & - & - \\
\hline & \multirow{2}{*}{4} & A & 0 & 0 & 0 & 0 & B1 & $\mathrm{B} 1,5$ & B1 & B2 & - & - & - & - \\
\hline & & B & 0 & B1 & 0 & B1,5 & - & B3 & B1 & B3 & - & - & - & - \\
\hline & \multirow{2}{*}{5} & A & 0 & B2 & 0 & B3 & - & $B 3,5$ & B1 & B4 & - & - & - & - \\
\hline & & B & 0 & B2 & B1 & B2 & B2 & B3,5 & B2 & B3,5 & T3 & T3 & T4 & $\mathrm{T} 4$ \\
\hline \multirow{10}{*}{ MEIO } & \multirow{2}{*}{1} & A & 0 & B1 & 0 & B2 & B1 & B3 & B1 & B3,5 & T2 & T4 & T4 & T4 \\
\hline & & B & 0 & B2, 5 & 0 & B3 & B1 & B3 & B2 & B4 & - & - & - & - \\
\hline & \multirow{2}{*}{2} & A & 0 & $\mathrm{~B} 1,5$ & 0 & $B 2,5$ & - & $\mathrm{B} 3,5$ & B1 & $\mathrm{B} 3,5$ & - & - & $\mathrm{T} 4$ & $\mathrm{~T} 4$ \\
\hline & & B & 0 & B1,5 & 0 & $\mathrm{~B} 2,5$ & - & B3 & B1 & B3 & - & - & $\mathrm{T} 4$ & $\mathrm{~T} 4$ \\
\hline & \multirow{2}{*}{3} & A & 0 & B3 & 0 & B3,5 & B1 & $B 3,5$ & B3 & $B 3,5$ & $\mathrm{~T} 2$ & $\mathrm{~T} 2$ & $\mathrm{~T} 4$ & $\mathrm{~T} 4$ \\
\hline & & B & 0 & B2 & B1 & B3 & B1 & $B 3,5$ & B2 & $\mathrm{B} 3,5$ & - & - & - & - \\
\hline & \multirow{2}{*}{4} & A & 0 & B1 & 0 & B2 & - & B2 & B1 & $\mathrm{B} 3,5$ & T1 & T1 & $\mathrm{T} 4$ & $\mathrm{~T} 4$ \\
\hline & & B & 0 & B1 & 0 & B2 & - & B3 & B1 & B3 & - & - & T4 & T4 \\
\hline & \multirow{2}{*}{5} & A & 0 & B1 & 0 & B2 & - & B1 & B3 & B4 & - & - & - & - \\
\hline & & B & 0 & 0 & 0 & 0 & - & B2 & - & B2 & - & - & - & - \\
\hline \multirow{10}{*}{ TOPO } & \multirow{2}{*}{1} & A & 0 & $B 2,5$ & 0 & B3 & - & B3 & - & $B 3,5$ & T1 & $\mathrm{T} 1$ & T4 & T4 \\
\hline & & B & 0 & B1,5 & 0 & B1,5 & B1 & $\mathrm{B} 1,5$ & B1 & $\mathrm{B} 1,5$ & T2 & $\mathrm{T} 2$ & T4 & T4 \\
\hline & \multirow{2}{*}{2} & A & 0 & B1 & 0 & B1,5 & - & $B 2,5$ & - & $B 2,5$ & - & - & - & - \\
\hline & & B & 0 & 0 & 0 & 0 & - & B1 & B1 & B2 & T3 & T3 & T4 & T4 \\
\hline & \multirow{2}{*}{3} & A & 0 & B1,5 & 0 & $\mathrm{~B} 2,5$ & - & B3 & B2 & B3 & - & - & - & - \\
\hline & & B & 0 & B1,5 & 0 & B3 & - & B3,5 & - & B3,5 & - & - & - & - \\
\hline & 4 & A & 0 & B1 & 0 & B1 & - & B1,5 & - & B2 & - & - & - & - \\
\hline & 4 & B & 0 & B1 & 0 & B2 & - & $B 2,5$ & - & $B 2,5$ & - & - & - & - \\
\hline & 5 & A & 0 & 0 & 0 & B1 & - & B1,5 & - & B2 & - & - & T4 & T4 \\
\hline & J & B & 0 & 0 & 0 & B2 & - & $B 2,5$ & - & B2, 5 & - & - & - & - \\
\hline
\end{tabular}

A= Pupunheira com espinho; $B=$ Pupunheira sem espinho; 0 = Ausência de ataque; B1a B1,9 = Ataque superficial de besouros; B2 a B2,9 = ataque moderado de besouros; B3 a B3,9 = ataque intenso de besouros; B4 = Disco totalmente atacado por besouros; T1 = ataque superficial de térmitas; $\mathrm{T} 2=$ ataque moderado de térmitas; $\mathrm{T} 3=$ Ataque intenso de térmitas; $\mathrm{T} 4=\mathrm{D}$ isco totalmente atacado por térmitas/Perda total da resistência; $(-)=$ ataque por outros organismos xilófagos.

N. tatarandae, eles observaram que esta espécie não demonstrou preferência por madeira sadia ou deteriorada.

Anoplotermes sp., que pertence à subfamília Apicotermitinae (Fontes, 1992), uma das mais primitivas dentro do grupo dos Termitidae (Krishna, 1970), também é comumente encontrada na região Amazônica, em florestas de terra firme e inundadas. É considerada de hábito intermediário, porque se alimenta de matéria orgânica do solo e madeira em estágio avançado de decomposição (Bandeira \& Macambira, 1988; Constantino, 1992; Martius,
1997). Segundo Souza \& Brown (1994), muitas espécies desse gênero são importantes consumidoras de matéria orgânica do solo.

Os besouros $D$. bifoveolatus e Minthea rugicollis, encontrados no ambiente urbano, pertencem ao grupo de insetos que se alimentam de açúcares e amido presentes em produtos vegetais, preferencialmente secos (Gray, 1972; Furnis \& Carolin, 1977). O fato da maior ocorrência $D$. bifoveolatus ter sido registrado nas amostras expostas na posição côncava pode ser explicado em função da umidade do disco nesta 


\section{ACTA \\ AMAZONICA}

DURABILIDADE NATURAL DO ESTIPE DE PUPUNHA

(Bactris gasipaes KUNTH, ARECACEAE) II. INSETOS posição ser menor, considerando que este inseto, apesar de atacar madeira levemente úmida, é comumente encontrado em madeiras secas (Flechtmann et al., 1996; 1997).

Hypothenemus eruditus e $X$. Ferrugineus, que foram registrados apenas no início do experimento, são comuns em árvores recém abatidas que volatilizam substâncias atrativas e que possuem umidade superior a 50\% (Beaver, 1976; Wood, 1982).

Assim como os cupins no ambiente florestal, o besouro D. bifoveolatus no ambiente urbano teve preferência pelas partes da base e do meio dos fustes da pupunha. Isto provavelmente está ligado ao comportamento alimentar desses insetos. Eles escolhem as partes das árvores mais ricas em alimento, o que deve ser o caso do meio e da base, principalmente, por possuírem maior quantidade de tecido xilemático. Como conseqüência, devem conter maiores quantidades de celulose, açucares e amido, alimentos preferenciais dos cupins e dos besouros.

Ainda na comparação do ataque das amostras da pupunheira em ambiente florestal e urbano, foi observado que, no ambiente florestal, o estipe foi deteriorado por três espécies de térmitas, em até 18 meses, enquanto que no ambiente urbano a deterioração no tempo de doze meses foi causada por besouros e pelo térmita $N$. similis, este inclusive sendo comum aos dois ambientes. Outro ponto comum nas amostras expostas a esses ambientes foi que somente uma pequena quantidade de discos, principalmente da madeira de cada variedade, não foi totalmente deteriorada pelos térmitas e besouros no decorrer do experimento. Diante deste resultado, a pupunheira com e sem espinhos pode ser classificada como não durável, pois, de acordo com a classificação de Cavalcante et al. (1982), sua vida útil ficou em torno de dois anos.

\section{CONCLUSÕES}

Neste experimento, o estipe de $B$. gasipaes (pupunha) foi considerado não durável, pois em ambiente florestal, sua vida útil foi limitada a 18 meses, ocasionado pelo ataque dos térmitas $H$. tenuis, Nasutitermes similis e $N$. tatarandae. Em ambiente urbano, o besouro $D$. bifoveolatus e o térmita $N$. similis foram responsáveis pela limitação da vida útil em doze meses.

Como as duas variedades da $B$. gasipaes apresentaram alta suscetibilidade ao ataque de besouros e cupins, quando expostas a ambientes florestal e urbano, recomenda-se que o estipe desta palmeira seja usado em ambiente interno, onde a umidade é menor. Neste ambiente, o material fica protegido das intempéries e, conseqüentemente, dos besouros e cupins que tem preferência por madeiras mais úmidas ou já deterioradas por fungos. Para sua utilização em ambiente externo, recomenda-se a aplicação de um produto preservante, para aumentar sua vida útil.

\section{AGRADECIMENTO}

Ao Dr. Basílio Frasco Vianez do INPA pelas sugestões ao texto do trabalho e aos técnicos de laboratório Frank Antônio de Oliveira Campos e Alfredo Pereira dos Santos pela coleta e triagem dos insetos.

\section{BIBLIOGRAFIA CITADA}

Abreu, R.L.S.; Bandeira, A.G. 1992. Besouros xilomicetófagos economicamente importantes da região de Balbina, Estado do Amazonas. Revista Árvore, 16(3): 346-356.

Abreu, R.L.S.; Silva, K.E.S. 2000. Resistência natural de dez espécies madeireiras da Amazônia ao ataque de Nasutitermes macrocephalus (Silvestri) e N. surinamensis (Holmgren) (Isoptera: Termitidae). Revista Árvore, 24(2): 229-234.

Araújo, J.C. 1991. Aspectos técnicos da implantação da cultura da pupunheira para produção de palmito. In: A Pupunbeira $e$ suas potencialidades econômicas. Secretaria de Produção Rural (SEPROR), Manaus, 37p.

Bandeira, A.G. 1989. Análise da termitofauna (Insecta: Isoptera) de uma floresta primária e de uma pastagem na Amazônia Oriental, Brasil. Bol. Mus. Para. Emílio Goeldi, sér. Zool., 5(2): 225-241.

Bandeira, A.G.; Macambira, M.L.J. 1988. Térmitas de Carajás, Estado do Pará, Brasil: Composição faunística, distribuição e hábito alimentar. Bol. Mus. Para. Emilio Goeldi, sér. Zool., 4(2): 175-190.

Bandeira, A.G.; Gomes, J.I.; Lisboa, P.L.B.; Souza, P.C.S. 1989. Insetos pragas de madeiras de edificações em Belém - Pará. EMBRAPA. Boletim de Pesquisa $\mathrm{n}^{\circ}$ 1, 24p.

Beaver, R. 1976. Biological studies of Brazilian Scolytidae and Platypodidae (Coleoptera). V. The tribe Xyleborini. Z. Ang. Ent., 80:15-30.

Cavalcante, M.S.; Montagna, R.G.; Lopes, G.A.C.; Mucci, F. 1982. Durabilidade de madeira em contato com o solo. II. Silvicultura em São Paulo. Rev Inst. Florestal, 16(2): 1383-1388p.

Clement, C.R. 1988. Domestication of the pejibaye palm (Bactris gasipaes): past and present. In: Balick, M.J. (Ed.). The Palm Tree of Life. Biology, Utilization and Conservation. Advances in Economic Botany, 6: 155-174.

Clement, C.R.; Mora Urpí, J. 1987. The pejibaye (Bactris gasipaes H.B.K.): Multi-use potential for the lowland humid tropics. Economic Botany, 41(2): 302-311.

Clement, C.R.; Arkcoll, D.B. 1991. The pejibaye (Bactris gasipaes H.B.K., Palmae) as an oil crop: potential and breeding strategy. Oleagineux, 46(7): 293-299.

Constantino, R. 1992. Abundance and diversity of térmites (Insecta:Isoptera) in two sites of primary rain forest in brasilian Amazonia. Biotropica, 24(3): 420-430.

Falesi, I.C. 1971. Solos do Distrito Agropecuário da SUFRAMA (Trecho: Km 30-Km 79, Rod. BR 174). Série Solos, (Supl. 1). IPEAM, Manaus, 99p.

Flechtmann, C.A.H.; Teixeira, E.P.; Gaspareto, C.L. 1996. Bostrichidae (Coleoptera) capturados em armadilhas iscadas com etanol em pinheiros tropicais na região de Agudos, SP. Revista do Instituto Florestal, 8(1): 17-44. 


\section{ACTA \\ AMAZONICA}

DURABILIDADE NATURAL DO ESTIPE DE PUPUNHA

(Bactris gasipaes KUNTH, ARECACEAE) II. INSETOS
Flechtmann, C.A.H.; Gaspareto, C.L.; Teixeira, E. 1997. Altura de vôo de Bostrichidae (Coleoptera) em Pinus caribaea v. hondurensis em P. Agudos, SP. Revista do Instituto Florestal, 9(1): 19-26.

Fontes, L.R. 1992. Key to the genera of New World Apicotermitinae (Isoptera: Termitidae). In Quintero, D; Aiello, A. (Eds.) Insects of Panama and Mesoamerica. Oxford University Press, Oxford. p. 242-248.

Furnis, R.L.; Carolin, V.M. 1977. Western forest insects. USDA, Miscellaneous Publication, $\mathrm{n}^{\circ}$ 1339. 654p.

Gomes, J.B.M. 1993. Crescimento e produção da pupunba (Bactris gasipaes Kunth) consorciada com mandioca (Manibot sculenta Carnaz), Urucum (Bixa orellana L.), banana pacovão (Musa paradiaca) e abacaxi (Ananas comosus) $L$. Dissertação de mestrado. Instituto Nacional de Pesquisas da Amazônia/Fundação Universidade do Amazonas. Manaus, Amazonas. 91p.

Gray, B. 1972. Economic tropical forest entomology. Annual Review Entomology, 17: 313-335.

INPA/CPPF. 1991. Catálogo de madeiras da Amazônia: Características tecnológicas; Área da Hidrelétrica de Balbina. CPPF/INPA, Manaus, 163p.
INPA/CPPF. 1993. Catálogo de Madeiras do Amapá: Características Tecnológicas. Manaus. INPA.

Jesus, M.A.; Morais, J.W.; Abreu, R.L.S; Cardias, M.F.C. 1998. Durabilidade natural de 46 espécies de madeiras amazônicas em contato com o solo em ambiente florestal. Scienta Forestalis, 54: 81-91.

Jesus, M.A.; Abreu, R.L.S. 2002. Durabilidade natural da madeira de pupunha (Bactris gasipaes Kunth). I. Fungos. Acta Amazonica, 32(4): 663-675.

Krishna, K. 1970. Taxonomy, phylogeny and distribution of termites. In: Krishna, K.; Weesner, F.M. (Eds.). Biology of termites. Vol. 2. Academic Press, New York. p. 127-153.

\author{
RECEBIDO EM 30/12/2002 \\ ACEITO EM 09/02/2004
}

